Check for updates

Cite this: Nanoscale Adv., 2019, 1 3009

\title{
In situ three-dimensional imaging of strain in gold nanocrystals during catalytic oxidation $\uparrow$
}

\author{
Ana Flavia Suzana, ${ }^{\text {ab }}$ Amélie Rochet, (D) *a Aline Ribeiro Passos, (D) a João Paulo Castro \\ Zerba, ${ }^{a}$ Carla Cristina Polo, ${ }^{a}$ Celso Valentim Santilli, ${ }^{\text {b }}$ Sandra Helena Pulcinelli, ${ }^{b}$ \\ Felisa Berenguer, ${ }^{c}$ Ross Harder, ${ }^{d}$ Evan Maxey ${ }^{d}$ and Florian Meneau (D) *a
}

Received 10th April 2019

Accepted 14th June 2019

DOI: 10.1039/c9na00231f

rsc.li/nanoscale-advances

The chemical properties of materials are dependent on dynamic changes in their three-dimensional (3D) structure as well as on the reactive environment. We report an in situ 3D imaging study of defect dynamics of a single gold nanocrystal. Our findings offer an insight into its dynamic nanostructure and unravel the formation of a nanotwin network under $\mathrm{CO}$ oxidation conditions. In situ/operando defect dynamics imaging paves the way to elucidate chemical processes at the single nano-object level towards defect-engineered nanomaterials.

\section{Introduction}

Metal nanoparticles used in heterogeneous catalysis present a large number of low-coordinated atoms where reactant adsorption and product desorption take place. These active sites are localised at corners, edges and surface defects. ${ }^{\mathbf{1 , 2}}$ The reactivity of the catalysts is tuned by the inherently strained surface structure, ${ }^{3,4}$ which is highly dynamic under reaction conditions. ${ }^{5-7}$ The improvement of chemical processes can be achieved through fine understanding of the evolution of strain and the formation of defects in both the surface and the inner core structure of the nanomaterials. Moreover, reaction conditions affect the behaviour of catalysts and their performance. In situ/operando studies under relevant and controlled environmental conditions must be performed. ${ }^{\mathbf{8 - 1 0}}$ However, in situ three-dimensional imaging techniques enabling nanometerresolution and strain determination simultaneously are lacking. To date, strain and defect information at the nanoscale are accurately obtained by electron microscopy ${ }^{\mathbf{1 1}, 12}$ with restrictions to reduced pressures due to the short penetration depth of electrons, preventing operando characterisation. On the other hand, powder X-ray diffraction in the hard X-ray regime enables the obtaining of strain structure data under operando

${ }^{a}$ Brazilian Synchrotron Light Laboratory (LNLS), Brazilian Center for Research in Energy and Materials (CNPEM), 13083-970, Campinas, SP, Brazil. E-mail: amelie. rochet@lnls.br; florian.meneau@lnls.br

${ }^{b}$ Instituto de Quimica, UNESP, Rua Professor Francisco Degni, 14800-900 Araraquara, SP, Brazil

'Synchrotron SOLEIL, L'Orme des Merisiers, BP48, Saint Aubin, 91192 Gif-sur-Yvette, France

${ }^{d}$ Advanced Photon Source, Argonne National Laboratory, 9700 South Cass Avenue, Argonne, IL 60439, USA

$\dagger$ Electronic supplementary information (ESI) available. See DOI: 10.1039/c9na00231f conditions but on a large assembly of particles, yielding solely averaged information. The availability of coherent hard X-rays from synchrotron light sources opens new opportunities for imaging studies of nanomaterials, ${ }^{\mathbf{1 3}, 14}$ where Bragg coherent Xray diffraction imaging (Bragg CDI) can reveal the strain distribution of single micro- and nano-sized objects in three dimensions and under operando conditions. ${ }^{15-21}$

Here, we investigate a model gold sample under CO oxidation reaction conditions at ambient pressure. Besides often being considered as a prototypical reaction to study the fundamental concepts of heterogeneous catalysis, this reaction is also of high environmental and societal importance. ${ }^{22}$ We use in situ Bragg X-ray coherent diffraction to unveil the defect dynamics and facetting process occurring on $120 \mathrm{~nm}$ gold (Au) nanoparticles supported on titanium dioxide $\left(\mathrm{TiO}_{2}\right)$. Although smaller gold nanoparticles are of greater interest in catalysis due to their catalytic performance at low temperature, ${ }^{7}$ the gold nanocrystal studied here presents both bulk and surface characteristics with defect sites, corners and edges with lowcoordinated atoms, similarly to smaller nanoparticles. Thus, our system can be considered as a model testing ground for surface restructuration that occurs under the industrially relevant reaction conditions used in this work. We describe the adsorbate-induced surface transformation, quantify the surface stress and further show that nanotwin formation plays a crucial role in the nanocrystal deformation mechanisms and correlates with the catalytic properties.

\section{Experimental section}

\section{Sample preparation}

The monodisperse gold nanoparticles (AuNPs) were prepared following the seeded-growth method. ${ }^{23}$ The seed nanoparticles were synthesised using the citrate reduction protocol. $0.5 \mathrm{~mL}$ of 
a $1 \%(\mathrm{w} / \mathrm{v})$ gold chloride solution in $50 \mathrm{~mL}$ of Milli-Q water was boiled up to $100{ }^{\circ} \mathrm{C}$. A $1 \%(\mathrm{w} / \mathrm{v})$ sodium citrate solution was added and the temperature maintained for five minutes under vigorous stirring. The seed and AuNP morphology and size were checked by scanning electron microscopy (Fig. S1†) and small angle X-ray scattering measurements (Fig. S2†). $50 \mathrm{~mL}$ of the AuNP colloidal dispersion was mixed with $100 \mathrm{mg}$ of $\mathrm{TiO}_{2}$ powder support and acidified with sulphuric acid to $\mathrm{pH}=1$. The theoretical loading of gold is $1 \mathrm{wt} \%$. The mixture was stirred for $2 \mathrm{~h}$ at room temperature (RT), centrifuged and washed until the supernatant became neutral. The powder was dried at $120^{\circ} \mathrm{C}$ for $6 \mathrm{~h}$, calcined at $300^{\circ} \mathrm{C}$ for $3 \mathrm{~h}$ and treated under a flux of pure $\mathrm{H}_{2}$ at $400{ }^{\circ} \mathrm{C}$ for $1 \mathrm{~h}$.

\section{Bragg coherent X-ray diffraction imaging}

The X-ray imaging experiments were performed at beamline 34ID-C of the Advanced Photon Source, USA. A $9 \mathrm{keV}$ focussed coherent X-ray beam $\left(500 \times 500 \mathrm{~nm}^{2}\right)$ was shined on isolated gold nanocrystals loaded in the operando cell. The same nanoparticle was measured in air at RT and $400{ }^{\circ} \mathrm{C}$ and then under reaction conditions at $\mathrm{RT}, 200$ and $400{ }^{\circ} \mathrm{C}$. A heating ramp of $3{ }^{\circ} \mathrm{C} \min ^{-1}$ was used to reach isothermal conditions for data acquisition. Under reaction conditions, a $\mathrm{CO} / \mathrm{O}_{2}$ gas mixture (CO : $\mathrm{O}_{2}, 0.5 \%: 3.7 \%$ ) was used with a total gas flow of 19.5 $\mathrm{mL} \min ^{-1}$.

The coherent diffraction patterns, (111) Bragg conditions, were collected with a Timepix detector, with $55 \times 55 \mu^{2}$ pixel sizes, placed $500 \mathrm{~mm}$ away from the sample. The 3D diffraction data were acquired as rocking curves with an angular step of $0.02^{\circ}$ and 61 frames of 5 second exposure, with 10 repetitions.

For the reconstruction, the phase retrieval was initiated with 20 error-reduction (ER) iterations, ${ }^{24}$ followed by 180 iterations of the hybrid input-output (HIO) algorithm, ${ }^{25}$ using a guided approach. ${ }^{26} 2220$ iterations were used for the reconstruction. The support constraint was refined using the shrink-wrap method and partial coherence was also taken into account. ${ }^{27}$ The 3D Bragg electron density and the 3D lattice displacement field projected along the [111] Q-vector were obtained by reconstructing the $3 \mathrm{D}$ diffraction data. Paraview (http:// www.paraview.org) was used to visualise the reconstructions in $2 \mathrm{D}$ and $3 \mathrm{D}$.

\section{Results and discussion}

Coherent X-ray diffraction patterns were measured around the (111) Bragg peak of a single nanocrystal, leading to a threedimensional Bragg peak (Fig. 1a). Applying iterative phase retrieval for coherent diffraction imaging, ${ }^{28}$ we reconstruct the three-dimensional (3D) Bragg electron density and lattice displacement field of the Au nanocrystal (Fig. 1b and c) with a spatial resolution close to $12 \mathrm{~nm}$ (Fig. S3†). The amplitude of the image obtained by Bragg CDI represents the electron density of the sample. The phase $\varphi(r)$ corresponds to the projection of the displacement of the crystal lattice $u(r)$, projected on the scattering vector $Q$ of the measured Bragg
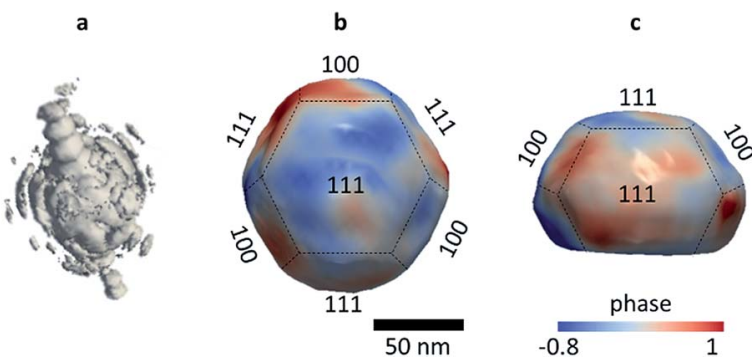

Fig. 1 (a) 3D diffraction pattern obtained by rocking scans around the (111) Bragg peak of a $120 \mathrm{~nm} \mathrm{Au} / \mathrm{TiO}_{2}$ nanoparticle in air at RT. The corresponding 3D reconstruction with a $30 \%$ isosurface seen from (b) top and (c) side views scaled with the phase. The facet orientations are shown.

diffraction peak, $\varphi(r)=Q u(r) \cdot{ }^{16}$ The presence of strains within a crystal is indicated by phase changes in the complex image.

Fig. $\mathrm{S} 4 \mathrm{a} \uparrow$ shows the $3 \mathrm{D}$ reconstruction of the Au nanocrystal in air at room temperature. The latter exhibits a truncated octahedral shape, with (100) and (111) facets (Fig. 1b and c). Upon $\mathrm{CO} / \mathrm{O}_{2}$ flow, the $3 \mathrm{D}$ reconstructions reveal the adsorbateinduced surface transformations occurring on the $\mathrm{Au}$ nanocrystal surface. While the pristine gold nanocrystal reconstruction (RT, air) exhibits a facetted morphology (in agreement with STEM images, Fig. S1 $\dagger$ ), it becomes smooth and rounded in a $\mathrm{CO} / \mathrm{O}_{2}$ environment at RT (Fig. S4b $\dagger$ ). These changes in surface morphology are in agreement with those observed under different environments for smaller nanoparticles. ${ }^{29,30}$ This indicates that the presence of $\mathrm{O}_{2}$ and $\mathrm{CO}$ gas molecules induces strong interactions between adsorbates and the surface atoms.

The phase shifts of the gold nanocrystal, in air and $\mathrm{CO} / \mathrm{O}_{2}$, are displayed as cross-sectional views in Fig. 2a (surrounded by an orange rectangle). A distinct phase redistribution is observed between the two gas environments: the reactive gas mixture $\mathrm{CO} /$ $\mathrm{O}_{2}$ induces a lattice displacement resulting in a smoother phase structure, evidencing the annihilation of several nanocrystal defects. The 3D strain distribution of the entire volume along the measurement direction was obtained by taking the derivative of the displacement field resulting from algorithmic phase reconstruction and is defined as $\partial u_{111}(r) / \partial x_{111}$, where $u_{111}(r)$ is the displacement field. Fig. $2 \mathrm{~b}$ and $\mathrm{c}$ display the $3 \mathrm{D}$ strain distribution of the nanocrystal and the cross sections of the strain field along [111], respectively. As for the phase distribution, the strain changes with the gas environment. Its highest values are concentrated at the periphery of the nanoparticle. We observe that a mean surface compressive to tensile strain swap occurs upon flowing the $\mathrm{CO} / \mathrm{O}_{2}$ gas mixture on the gold nanocrystal surface. The average surface strain in air equals to -1.7 $\times 10^{-4}$ and becomes tensile with a mean value of $1.1 \times 10^{-5}$ under $\mathrm{CO} / \mathrm{O}_{2}$.

This pattern of displacement observed on the surface of the nanocrystal under $\mathrm{CO} / \mathrm{O}_{2}$ can be attributed to the decrease of the surface stress, due to the annihilation of point defects. The surface stress $\sigma_{\mathrm{s}}$ can be estimated (ESI $\dagger$ ) using the YoungLaplace equation. ${ }^{31}$ At the position indicated by the grey arrow 


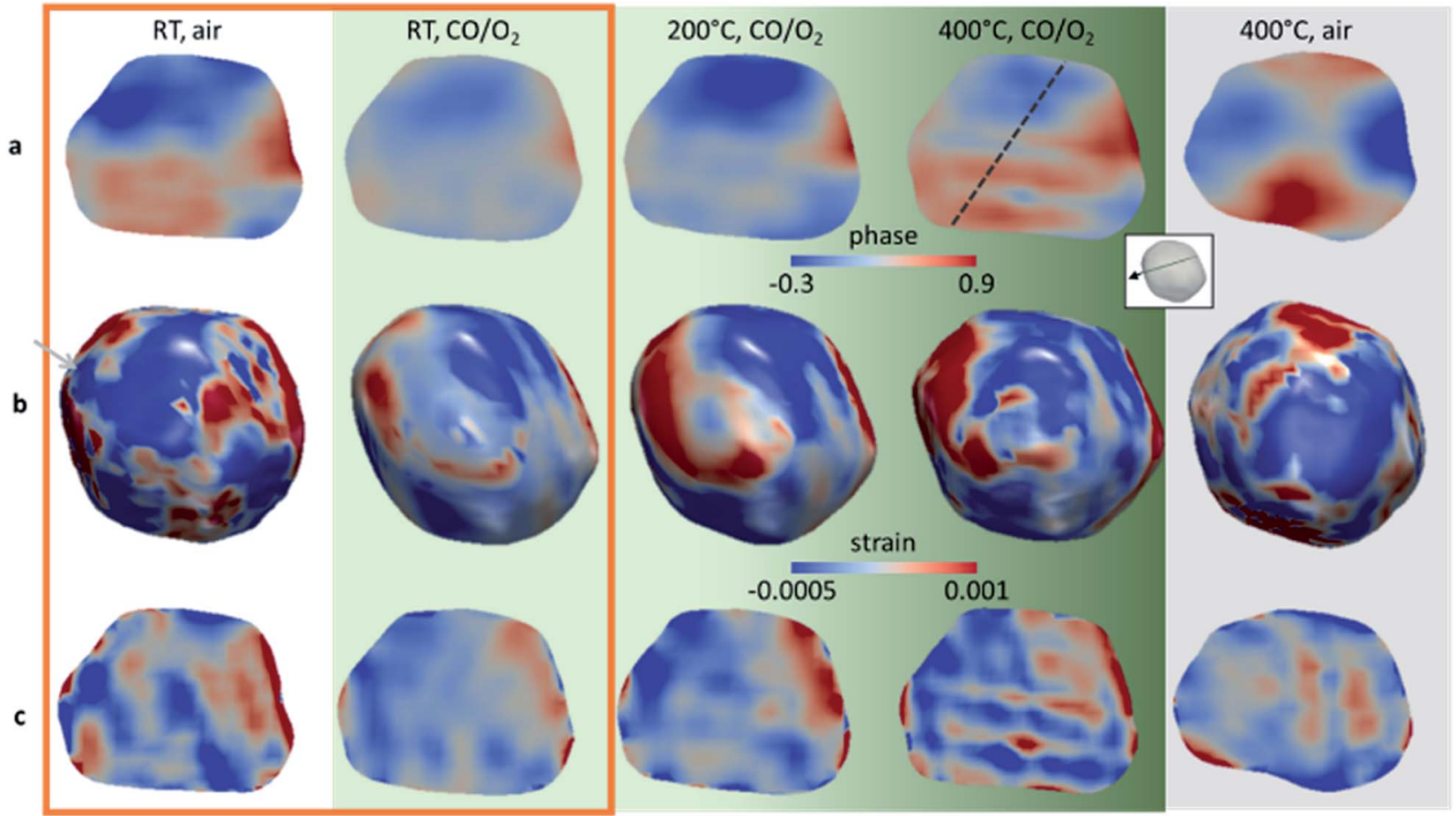

Fig. 2 Distribution of the phase and strain field projected along the (111) direction of the same $\mathrm{Au} / \mathrm{TiO} \mathrm{O}_{2}$ nanoparticle in air, at RT and $400{ }^{\circ} \mathrm{C}$ and under $\mathrm{CO} / \mathrm{O}_{2}$ at RT, $200{ }^{\circ} \mathrm{C}$ and $400{ }^{\circ} \mathrm{C}$. Particle cross sections of the inner structure of the nanoparticle corresponding to the atomic displacement (a) and the strain field projected along (111) (c). (b) 3D strain distribution of the strain field projected along (111) from the top view. While the green gradient illustrates the increase of catalytic activity with temperature, the orange rectangle emphasises the RT conditions and the grey background the state at $400^{\circ} \mathrm{C}$ in air. In the inset, the green surface indicates the position of the cross section presented in (a) and (c) and the black arrow the orientation of the [111] Q-vector. The dashed black line in (a) shows the position of the line scan presented in Fig. 4. Animations showing the 3D reconstructed Bragg electron density and all cross sections along two axes of the gold nanocrystal, under $\mathrm{CO} / \mathrm{O}_{2}$ at RT and $400{ }^{\circ} \mathrm{C}$, are available in ESI Movies S1 and S2. $\dagger$

in Fig. 2b, the surface stress of the nanocrystal in air is $2.4 \pm$ $0.3 \mathrm{~N} \mathrm{~m}^{-1}$, typical of tensile surface stress of metals in the range of $2 \mathrm{~N} \mathrm{~m}^{-1} \cdot{ }^{31,32}$ On the other hand, the surface stress at the same position under $\mathrm{CO} / \mathrm{O}_{2}$ turns out to be compressive and equals to $-1.7 \pm 0.1 \mathrm{~N} \mathrm{~m}^{-1}$. The adsorbate-induced surface stress on the gold nanocrystal leads to a compressive stress change at RT of $-4.1 \mathrm{~N} \mathrm{~m}^{-1}$, resulting in the inward displacement of the curved regions. The nanocrystal surface modification is directly influenced by the surface stress, modifying the proportion of undercoordinated sites and tuning the catalytic performance of the nanocrystals. Although effects induced by the presence of the support cannot be excluded, considering the size of the nanoparticle, the metal-support interface is negligible in comparison with the total Au surface.

Moreover, we succeeded in capturing in situ the surface restructuration of the same nanoparticle under $\mathrm{CO} / \mathrm{O}_{2}$ at $200{ }^{\circ} \mathrm{C}$ and $400{ }^{\circ} \mathrm{C}$. Though gold particles that are of interest for catalysis are smaller, we observed moderate catalytic activity (Fig. S5 $\dagger$ ) for our model sample loaded inside our in situ imaging cell. ${ }^{33}$ The catalyst presents an increased activity at $400{ }^{\circ} \mathrm{C}$ compared to $200{ }^{\circ} \mathrm{C}$, and none at RT. Fig. $2 \mathrm{~b}$ shows the three-dimensional strain field distribution of the same nanocrystal at 200 and $400{ }^{\circ} \mathrm{C}$ under $\mathrm{CO} / \mathrm{O}_{2}$, revealing the nanocrystal surface dynamics. It is worth mentioning that $200{ }^{\circ} \mathrm{C}$ is higher than the Hüttig temperature $\left(T_{\mathrm{H}}=127^{\circ} \mathrm{C}\right)$, above which the mobility of surface atoms becomes significant and $400{ }^{\circ} \mathrm{C}$ is slightly above the Tammann temperature $\left(T_{\mathrm{T}}=327^{\circ} \mathrm{C}\right)$, above which the mobility of the crystal lattice becomes significant and solid-state diffusion takes place. ${ }^{34}$ At the highest temperature, we evidenced the reconstitution of sharper edges and corners. As previously stated, nanocrystal corners and edges are regions of low-coordinated atoms and the location of the active sites. A direct correlation between the surface modifications of the nanocrystal and its functional properties is uniquely unveiled here.

Furthermore, under working conditions, the surface modifications are also accompanied by a one-fold increase of the average surface strain, reaching $1.35 \times 10^{-4}$ and with corner regions of surface stress $>6 \mathrm{~N} \mathrm{~m}^{-1}$. This is in agreement with "dband center theory". ${ }^{35}$ The increase of tensile strain strengthens the metal-adsorbate bond by shifting the d-band center toward the Fermi level and leads to improved catalytic activity. At the same time, strong lattice dynamics in the core structure of the nanocrystal, along with anisotropic strain patterns, are observed (Fig. 2). The temperature increase up to $200{ }^{\circ} \mathrm{C}$ is accompanied by a phase reorganisation with highly distorted lattice regions (Fig. 3) localised and concentrated at the surface of three edges of the nanoparticle. Edges and steps on the 

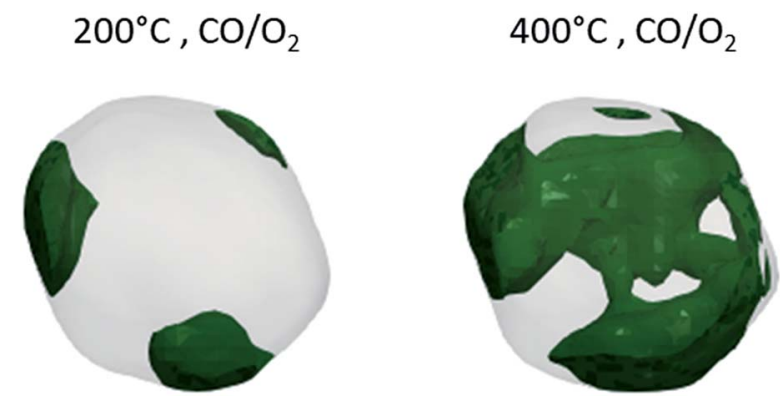

Fig. 3 Distribution of the maximum phase shift [0.4-1.1 rad] of the same $\mathrm{Au} / \mathrm{TiO}_{2}$ nanoparticle under $\mathrm{CO} / \mathrm{O}_{2}$ at $200{ }^{\circ} \mathrm{C}$ and $400{ }^{\circ} \mathrm{C}$ from the bottom view. The crystal at $200^{\circ} \mathrm{C}$ shows highly distorted areas concentrated at the surface of three edges. At $400{ }^{\circ} \mathrm{C}$, a complex network is present with highly distorted areas crossing the entire volume of the crystal and connecting the three regions highlighted at $200{ }^{\circ} \mathrm{C}$.

surface of the nanoparticle, rather than the facets, are known to be preferential sites for $\mathrm{CO}$ molecule adsorption. ${ }^{30}$ It is worth mentioning that $200{ }^{\circ} \mathrm{C}$ corresponds to a temperature just below the onset temperature of the conversion of $\mathrm{CO}$ into $\mathrm{CO}_{2}$ for this sample (Fig. S5 $\dagger$ ), confirming that the lattice reorganisation is a driven force building the active sites. At $400{ }^{\circ} \mathrm{C}$, a network of highly distorted lattices connects those three edges (Fig. 3).

The phase evolution between the nanocrystal at RT and its active state at $400{ }^{\circ} \mathrm{C}$ is emphasised in the line scan (Fig. 4), the latter exhibiting an oscillatory behaviour. Each phase oscillation corresponds to an atomically defective domain, spanning across the nanocrystal and forming a network of parallel stripes with strained interfaces. These defects enable accommodation of the tensile strain which builds up within the nanocrystal and is attributed to a nanotwin network. Indeed, twin domains are planar defects comprising at least two adjacent stacking faults.

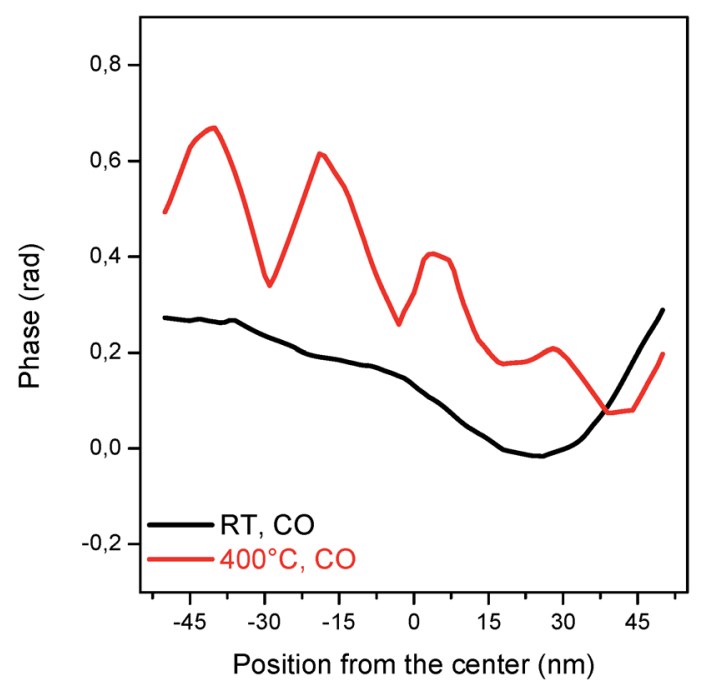

Fig. 4 Line scans of the phase at RT (black) and $400{ }^{\circ} \mathrm{C}$ (red) under $\mathrm{CO} / \mathrm{O}_{2}$. The line scan corresponds to the values of the position of the dashed line shown (for $400^{\circ} \mathrm{C}$ ) in Fig. $2 \mathrm{a}$.
They occur when a stacking sequence of planes share one of these planes and follow a mirrored packing order. ${ }^{36}$ Twin domains should be "invisible" in the retrieved Bragg electron density maps since the twin crystal does not satisfy the Bragg condition. ${ }^{36}$ The phase offset between the parent crystals should be proportional to the twin width, although the twin boundary interfaces as well as the strain contribute to the phase variations, explaining the lower phase offset observed here. The nanotwins cannot be faithfully reconstructed since they are smaller than the resolution achieved here, but the network and its positions are indeed resolved to a much higher precision (Fig. S6 $\dagger$ ). It is important to note that this complex phase structure is not observed at $400{ }^{\circ} \mathrm{C}$ in air (Fig. 2, grey background and line scan Fig. $\mathrm{S} 7 \dagger$ ), emphasising that the defect formation is not solely induced and controlled by the temperature.

Therefore the nanotwins play a crucial role in the deformation mechanism, facetting process and catalytic behaviour of the gold nanocrystals. The large positive surface stress is the trigger to the surface reconstruction through a glide motion. According to dislocation theory, ${ }^{37}$ glide motion is performed by the movement of a dislocation or the formation of two partial dislocations (Shockley partials), the latter being energetically more favourable than a single dislocation in the case of FCC metals. When surface stress is applied, Shockley partial dislocations are emitted, leading to twin nucleation which propagates across the nanocrystal. In fact, the highly distorted regions observed at $200^{\circ} \mathrm{C}$ (Fig. 3) are likely the nucleation sites of the Shockley partials leading to the twin nucleation which propagates across the core of the nanocrystal between 200 and $400{ }^{\circ} \mathrm{C}$.

\section{Conclusions}

This work reports the first in situ 3D imaging of strain on a gold supported nanoparticle under $\mathrm{CO}$ oxidation conditions. We use Bragg CDI to image a gold nanocrystal at ambient pressure and reach a spatial resolution of $12 \mathrm{~nm}$, which is not attainable with other techniques. We observe and quantify the adsorbateinduced surface stress on the gold nanocrystal leading to surface restructuration. Furthermore, the present study reveals the role of the nanotwin network in the deformation of the gold nanostructure and sheds further light on the defect dynamics responsible for its chemical properties. Considering the size of the nanoparticle, the metal-support interface is negligible in comparison with the total gold surface, but the effects of the support on the defective domains would deserve further investigation.

With the advent of upgraded and fourth-generation synchrotron sources based on a multi-bend achromat lattice like the SIRIUS facility, ${ }^{38}$ the coherent flux available in the hard$\mathrm{X}$-ray regime can be expected to increase by two orders of magnitude in the near future. ${ }^{39}$ This will directly reduce the total data acquisition time, and enable performing timeresolved operando imaging and reaching close to atomic resolution. $^{40}$ Improving the time-resolution will allow a more detailed analysis of the transient states and could potentially 
help to improve real world catalysts through a more comprehensive description of the mechanism of defect formation correlated with the active sites.

\section{Author contributions}

F. M. and A. R. conceived the project. F. M., A. F. S., E. M. and R. $\mathrm{H}$. carried out the Bragg CDI imaging experiments at the 34-IDC beamline. A. F. S. prepared the gold on $\mathrm{TiO}_{2}$ samples and performed with A. R. and A. R. P. the materials characterisation. F. M., A. F. S., A. R., A. R. P., J. P. Z., C. C. P. and R. H. analysed the imaging experiments. F. M., A. R., A. F. S. and A. R. P. wrote the paper with input from all authors.

\section{Conflicts of interest}

There are no conflicts to declare.

\section{Acknowledgements}

The Advanced Photon Source (APS) is acknowledged for providing beamtime at the 34-ID-C beamline (proposal GUP53802). The SAXS1 beamline from the LNLS and the HighPerformance Computing system are acknowledged (proposals 20150205 and 20180025). The authors would like to thank the LNNano for technical support during electron microscopy work (proposal SEM-21178). This research used resources of the Advanced Photon Source, a U.S. Department of Energy (DOE) Office of Science User Facility operated for the DOE Office of Science by Argonne National Laboratory under Contract No. DEAC02-06CH11357. This work was supported by the Brazilian funding agency FAPESP (2014/25964-5, 2014/27127-3, 2017/ 23050-4, and 2018/08816-3).

\section{Notes and references}

1 S. N. Rashkeev, A. R. Lupini, S. H. Overbury, S. J. Pennycook and S. T. Pantelides, Phys. Rev. B: Condens. Matter Mater. Phys., 2007, 76, 035438.

2 C. Y. Wu, W. J. Wolf, Y. Levartovsky, H. A. Bechtel, M. C. Martin, F. D. Toste and E. Gross, Nature, 2017, 541, 511.

3 M. Mavrikakis, B. Hammer and J. K. Nørskov, Phys. Rev. Lett., 1998, 81, 2819.

4 A. Kuwabara, E. D. Boyes, M. L. Pay, P. L. Gai, M. J. Walsh and K. Yoshida, Nano Lett., 2012, 12, 2027.

5 S. B. Vendelbo, C. F. Elkjær, H. Falsig, I. Puspitasari, P. Dona, L. Mele, B. Morana, B. J. Nelissen, R. Van Rijn, J. F. Creemer, P. J. Kooyman and S. Helveg, Nat. Mater., 2014, 13, 884.

6 K. F. Kalz, R. Kraehnert, M. Dvoyashkin, R. Dittmeyer, R. Gläser, U. Krewer, K. Reuter and J. D. Grunwaldt, ChemCatChem, 2017, 9, 17.

7 Y. He, J.-C. Liu, L. Luo, Y.-G. Wang, J. Zhu, Y. Du, J. Li, S. X. Mao and C. Wang, Proc. Natl. Acad. Sci. U. S. A., 2018, 115, 7700 .

8 M. G. O'Brien, A. M. Beale, S. D. M. Jacques, M. Di Michiel and B. M. Weckhuysen, Appl. Catal., A, 2011, 391, 468.
9 Y. Li, D. Zakharov, S. Zhao, R. Tappero, U. Jung, A. Elsen, P. Baumann, R. G. Nuzzo, E. A. Stach and A. I. Frenkel, Nat. Commun., 2015, 6, 7583.

10 F. Meirer and B. M. Weckhuysen, Nat. Rev. Mater., 2018, 3, 324. 11 C. L. Johnson, E. Snoeck, M. Ezcurdia, B. RodríguezGonzález, I. Pastoriza-Santos, L. M. Liz-Marzán and M. J. Hÿtch, Nat. Mater., 2008, 7, 120.

12 B. Goris, J. De Beenhouwer, A. De Backer, D. Zanaga, K. J. Batenburg, A. Sánchez-Iglesias, L. M. Liz-Marzán, S. Van Aert, S. Bals, J. Sijbers and G. Van Tendeloo, Nano Lett., 2015, 15, 6996.

13 S. Baier, C. D. Damsgaard, M. Scholz, F. Benzi, A. Rochet, R. Hoppe, T. Scherer, J. Shi, A. Wittstock, B. Weinhausen, J. B. Wagner, C. G. Schroer and J.-D. Grunwaldt, Microsc. Microanal., 2016, 22, 178.

14 A. M. Wise, J. N. Weker, S. Kalirai, M. Farmand, D. A. Shapiro, F. Meirer and B. M. Weckhuysen, ACS Catal., 2016, 6, 2178.

15 M. A. Pfeifer, G. J. Williams, I. A. Vartanyants, R. Harder and I. K. Robinson, Nature, 2006, 442, 63.

16 I. Robinson and R. Harder, Nat. Mater., 2009, 8, 291.

17 M. Watari, R. A. McKendry, M. Vögtli, G. Aeppli, Y. A. Soh, X. Shi, G. Xiong, X. Huang, R. Harder and I. K. Robinson, Nat. Mater., 2011, 10, 862.

18 D. Kim, M. Chung, J. Carnis, S. Kim, K. Yun, J. Kang, W. Cha, M. J. Cherukara, E. Maxey, R. Harder, K. Sasikumar, S. K. R. S. Sankaranarayanan, A. Zozulya, M. Sprung, D. Riu and H. Kim, Nat. Commun., 2018, 9, 3422.

19 A. Yau, W. Cha, M. W. Kanan, G. B. Stephenson and A. Ulvestad, Science, 2017, 356, 739.

20 U. Ulvestad, A. Singer, J. N. Clark, H. M. Cho, J. W. Kim, R. Harder, J. Maser, Y. S. Meng and O. G. Shpyrko, Science, 2015, 348, 1344.

21 J. Ihli, J. N. Clark, N. Kanwal, Y.-Y. Kim, M. A. Holden, R. J. Harder, C. C. Tang, S. E. Ashbrook, I. K. Robinson and F. C. Meldrum, Chem. Sci., 2019, 10, 1176.

22 H. J. Freund, G. Meijer, M. Scheffler, R. Schlögl and M. Wolf, Angew. Chem., Int. Ed., 2011, 50, 10064.

23 S. Huo, H. Ma, K. Huang, J. Liu, T. Wei, S. Jin, J. Zhang, S. He and X. J. Liang, Cancer Res., 2013, 73, 319.

24 R. W. Gerchberg and W. O. Saxton, Optik, 1972, 35, 237.

25 J. R. Fienup, Appl. Opt., 1982, 21, 2758.

26 C.-C. Chen, J. Miao, C. W. Wang and T. K. Lee, Phys. Rev. B: Condens. Matter Mater. Phys., 2007, 76, 064113.

27 S. Marchesini, H. He, H. N. Chapman and S. P. Hau-Riege, Phys. Rev. B: Condens. Matter Mater. Phys., 2003, 68, 140101.

28 J. Miao, P. Charalambous, J. Kirz and D. Sayre, Nature, 1999, 400, 342.

29 T. Uchiyama, H. Yoshida and N. Kamiuchi, Surf. Sci., 2017, 659, 16.

30 Y. Kuwauchi, H. Yoshida, T. Akita, M. Haruta and S. Takeda, Angew. Chem., Int. Ed., 2012, 51, 7729.

31 I. Robinson, J. Phys. Soc. Jpn., 2013, 82, 1.

32 W. Haiss, Rep. Prog. Phys., 2001, 64, 591.

33 A. Rochet, A. F. Suzana, A. R. Passos, T. Kalile, F. Berenguer, C. V. Santilli, S. H. Pulcinelli and F. Meneau, Catal. Today, 2018, DOI: 10.1016/j.cattod.2018.12.020. 
34 Y. Odarchenko, D. J. Martin, T. Arnold and A. M. Beale, Faraday Discuss., 2018, 208, 243.

35 J. Sun, F. Wang, Y. Liu, Y. Ni, H. Zhou, C. F. Guo and S. Chen, $R S C A d v .$, 2017, 7, 22479.

36 A. Ulvestad, J. N. Clark, R. Harder, I. K. Robinson and O. G. Shpyrko, Nano Lett., 2015, 15, 4066.
37 D. Hull and D. J. Bacon, Introduction to dislocations, Oxford, 5th edn, 2011.

38 L. Liu, N. Milas, A. H. C. Mukai, X. R. Resende and F. H. de Sá, J. Synchrotron Radiat., 2014, 21, 904.

39 M. Yabashi and H. Tanaka, Nat. Photonics, 2017, 11, 12.

40 S. H. Dietze and O. G. Shpyrko, J. Synchrotron Radiat., 2015, 22, 1498. 